Cite this: Phys. Chem. Chem. Phys., 2013, 15, 11696

\title{
Individual dispersion of carbon nanotubes in epoxy via a novel dispersion-curing approach using ionic liquids $†$
}

\author{
Nishar Hameed, ${ }^{* a}$ Nisa V. Salim, ${ }^{a}$ Tracey L. Hanley, ${ }^{b}$ Mrunali Sona, ${ }^{a}$ Bronwyn L. Fox ${ }^{a}$ \\ and Qipeng Guo*a
}

The effective dispersion of carbon nanotubes (CNTs) in a thermoset was achieved using ionic liquid as the dispersion-curing agent. We preferentially dispersed multiwalled carbon nanotubes (MWCNTs) down to individual tube levels in epoxy resin. Here the dispersion is ruled by the depletion of physical bundles within the MWCNT networks, for which molecular ordering of ionic liquids is considered responsible. The quantitative analyses using ultra small angle X-ray scattering (USAXS) confirmed the dispersion of individual MWCNTs in the matrix. The distance between the dispersed nanotubes was calculated at different nanotube loadings using the power law fitting of the USAXS data. The fine

Received 8th January 2013,

Accepted 10th May 2013

DOI: $10.1039 / \mathrm{c} 3$ cp00064h

www.rsc.org/pccp dispersion and subsequent curing, both controlled by ionic liquid, lead to composites with substantially enhanced fracture mechanical and thermomechanical properties with no reduction in thermal properties. Merging processing techniques of nanocomposites with ionic liquid for efficient dispersion of nanotubes and preferential curing of thermosets facilitates the development of new, high performance materials.

\section{Introduction}

Development of high performance materials by the incorporation of carbon nanotubes (CNTs) into polymer matrices has been difficult because thermodynamic and kinetic barriers inhibit the effective dispersion of nanotubes. The incorporation and uniform dispersion of carbon nanotubes in epoxy thermosets could facilitate engineers to create materials that potentially compete with the most advanced materials in nature. ${ }^{1}$ The unique combination of outstanding mechanical, thermal, and electrical properties of CNTs makes them excellent nanofillers for the fabrication of advanced materials. Successful enhancement in mechanical and electrical properties via reinforcement is expected only when the nanofillers are well dispersed within the polymer matrix. Moreover, the orientation, as well as the CNT/matrix interfacial strength, also determines

\footnotetext{
${ }^{a}$ Institute for Frontier Materials, Deakin University, Locked Bag 2000, Geelong, Victoria 3220, Australia. E-mail: nishar.hameed@deakin.edu.au, qguo@deakin.edu.au

${ }^{b}$ Institute Materials Engineering, Australian Nuclear Science and Technology Organisation, Lucas Heights, New South Wales 2234, Australia

$\dagger$ Electronic supplementary information (ESI) available: Ionic liquid optimization, $T_{\mathrm{g}}$-composition plots, DSC thermograms and thermogravimetric curves. See DOI: $10.1039 / \mathrm{c} 3 \mathrm{cp} 00064 \mathrm{~h}$
}

the effective physical properties of these materials. ${ }^{2-5}$ However, CNTs, having a high aspect ratio and a large $\pi$-electronic surface, typically assemble to give bundles which are heavily entangled with one another. The common dispersing methods such as solution mixing for the preparation of nanocomposites are a concern as CNTs repel most polar and nonpolar solvents. ${ }^{6}$ Surface functionalization has been used to improve the dispersion of CNTs, ${ }^{7,8}$ however it deteriorates the intrinsic properties of these fillers except for some very recent studies. ${ }^{9}$ Therefore, the dispersion of CNTs down to an individual tube level in polymers remains a great challenge and despite numerous advances, polymer-CNTs composites have yet to demonstrate the expected property improvements.

Fukushima et al., achieved a superior dispersion of CNTs in room temperature ionic liquid (IL) by mixing single walled carbon nanotubes (SWCNTs) with 1-butyl-3-methylimidazolium tetrafluoroborate $\left(\mathrm{BMIMBF}_{4}\right) .{ }^{10}$ These SWCNT-IL mixtures or 'bucky gels' are highly stable and the nanotubes are finely dispersed due to the orientation of CNTs in IL by possible 'imidazolium cation $-\pi$ ' interactions. ${ }^{11}$ Generally CNTs adhere tightly to each other due to strong $\pi-\pi$ stacking interactions, making it very difficult to disperse by common solvents. When CNTs and IL mixed together the shear force detaches the nanotubes from the bundles and the tubes are then 
wrapped by $\mathrm{IL}^{12}$ It is proposed that the $\pi-\pi$ stacking interactions of nanotubes are shielded by the high dielectric constants of IL restricting them from rebundling. Moreover it is found that IL can disperse a higher concentration of CNTs than surfactants or DNA. ${ }^{13}$ This molecular ordering and interaction may initiate the dispersion of CNTs in ILs and high performance polymer based composites can be efficiently prepared by taking advantage of a strong affinity of the imidazolium ion toward the $\pi$-electronic nanotube surface. In another important study, ILs were used as thermally latent initiators for polymerization of epoxy resins. ${ }^{14,15}$ BMIMBF $_{4}$ and recently, 1-ethyl-3methylimidazolium dicyanamide $\left[\operatorname{EmiN}(\mathrm{CN})_{2}\right]$ have been successfully used as the hardener for epoxy resins. The ionic liquid is incorporated into the polymer during curing and forms a cross-linked epoxy network with bulky counterions. ${ }^{15-18}$

In this work, we present an investigation of highly dispersed multiwalled carbon nanotubes (MWCNTs) in epoxy by combining the outstanding dispersing and hardening efficiency of ILs. The ionic liquid, $\mathrm{BMIMBF}_{4}$, was used to disperse MWCNTs in epoxy and then composites with superior mechanical properties were prepared. In other words $\mathrm{BMIMBF}_{4}$ acts as both a dispersing agent for MWCNTs and a curing initiator for epoxy resin. In the final material, $\mathrm{BMIMBF}_{4}$ becomes a part of the crosslinked matrix. Here, the cost advantage of MWCNTs over SWCNTs and dispersing potential of $\mathrm{BMIMBF}_{4}$ over other ILs can provide an alternative route for the preparation of new functional materials. Recent studies of epoxy nanocomposites try to achieve maximum properties at low filler concentrations to make them cost-effective and therefore, composites at low nanotube loadings were investigated. Moreover, the cost of IL is also equitable considering the relatively small fraction of ILs used here as an additive. The whole process has thus a potential to make cost-effective composites to be used in aerospace and automotive applications.

\section{Results and discussion}

Epoxy suspensions and composites containing 0.05 to $1 \mathrm{wt} \%$ of MWCNTs were prepared using the IL dispersion-curing method. The MWCNT dispersion capacity of ionic liquids in epoxy was verified using USAXS techniques. Epoxy-MWCNT suspensions containing $0.5 \% \mathrm{BMIMBF}_{4}$ were used to analyze the dispersion properties. A dedicated analysis of USAXS data at various $q$ regions enables us to quantitatively demonstrate the rigid rod behavior and crossover network behavior of viscous CNT solutions and their cured composites. The level of dispersion was measured first in viscous suspensions of epoxy + $\mathrm{BMIMBF}_{4}+\mathrm{MWCNTs}$ prior to curing. We have physically observed that, below $0.5 \mathrm{wt} \%$ of MWCNT concentration, the suspensions flow freely under the influence of gravity and above this point, the suspensions show physical gel behavior as shown in Fig. $1 .{ }^{19}$ This gelation implies that further dispersion is achievable, however eventually network formation will limit the degree of dispersion that could be achieved. The gelation is apparently due to the formation of networks owing to the overlapping of individual MWCNTs or their bundles


Q

Fig. 1 Photographs of MWCNT suspensions in epoxy $+\mathrm{BMIMBF}_{4}$ mixtures. The concentration of $\mathrm{BMIMBF}_{4}$ is $0.5 \mathrm{wt} \%$ to epoxy. (a) Epoxy $+\mathrm{BMIMBF}_{4}$ mixture without MWCNTs, flows slowly when turn upside down. (b) Suspension containing $0.2 \mathrm{wt} \%$ MWCNTs. (c) Suspension containing $0.5 \mathrm{wt} \%$ MWCNTs forms a physical gel. (d) Possible structure of MWCNT dispersion in suspensions, $\xi$ is the crossover point that gives the average distance between the intersections of the crosslinked network. (e) The typical scattering profile model illustrating the crossover point in a suspension. ${ }^{19}$

depending on the dispersion state (Fig. 1d). The average distance between the two neighboring contact points in the network is the mesh size $(\xi) .{ }^{19}$ The concentration dependence of $\xi$ can be determined from SAXS intensity profiles to quantify the network crossover effect. The $\xi$ value is expected to decrease with increasing CNT concentrations.

CNTs can be considered as rigid rods and scattering intensity from stable solutions of individual CNTs or rod-like CNT bundles is expected to exhibit a $Q^{-1}$ intensity variation. ${ }^{20}$ On the other hand weakly dispersed CNT solutions show $Q^{-2}$ variation in scattering intensity. The USAXS scattering profiles of MWCNT suspensions stabilized by $\mathrm{BMIMBF}_{4}$ and epoxy are shown in Fig. 2. The upturn in scattering for samples containing MWCNTs at $Q<0.01 \mathrm{~A}^{-1}$ is due to the presence of dispersed nanotubes. ${ }^{21}$ The rigid rod $\left(Q^{-1}\right)$ and network structure $\left(Q^{-2}\right)$ contributions are highlighted in each figure and $Q_{c}$ represents the crossover point. In the USAXS profiles the low $Q$ scattering is due to the MWCNTs dispersed in the solution and it clearly demonstrates the power law behavior. The intermediate scattering that arises from the epoxy $+\mathrm{BMIMBF}_{4}$ mixture and the higher flat $Q$ region is the incoherent scattering. Considering distinct scattering patterns, the total scattering intensity can be divided into three parts namely; power law MWCNT scattering, solvent scattering from the epoxy $+\mathrm{BMIMBF}_{4}$ mixture and the incoherent background scattering.

$$
\frac{\mathrm{d} \Sigma}{\mathrm{d} \Omega}(Q)=\left.\frac{\mathrm{d} \Sigma}{\mathrm{d} \Omega}\right|_{\mathrm{MWCNT}}+\left.\frac{\mathrm{d} \Sigma}{\mathrm{d} \Omega}\right|_{\mathrm{EPO}+\mathrm{IL}}+I_{\mathrm{inc}}=\frac{I_{0}}{Q^{\alpha}}+I_{\mathrm{EPO}+\mathrm{IL}}+I_{\mathrm{inc}}
$$

where $I_{0}$ is the coefficient of the power law, $\alpha$ is the exponent and the coherent scattering from the solution and incoherent scattering of the background are given by $I_{\mathrm{EPO}+\mathrm{IL}}$ and $I_{\mathrm{inc}}$ respectively. The incoherent background was taken as the flat 

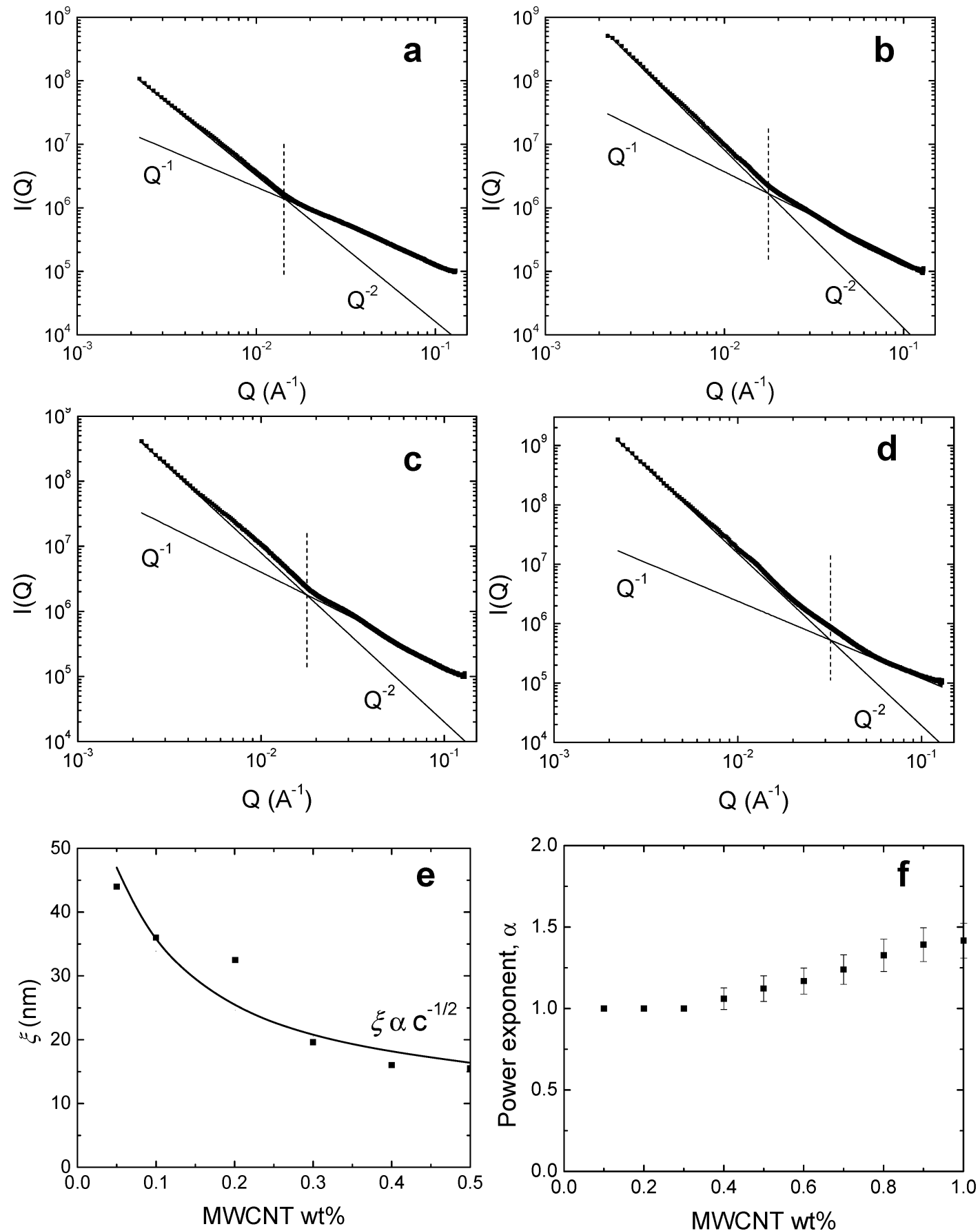

Fig. 2 USAXS scattering profiles of epoxy $+\mathrm{BMIMBF}_{4}+\mathrm{MWCNT}$ suspensions at low nanotube concentrations. The concentration of $\mathrm{BMIMBF}_{4}$ was kept constant at $0.5 \mathrm{wt} \%$ of epoxy. (a) $0.05 \mathrm{wt} \%$, (b) $0.1 \mathrm{wt} \%$, (c) $0.2 \mathrm{wt} \%$, and (d) $0.3 \mathrm{wt} \%$ of MWCNTs. The solid lines in figures (a-d) represent the best fit values to eqn (1) in the text and the vertical dotted lines represent the crossover points. (e) The variation in mesh size ( $\xi)$ with concentration of MWCNTs. The solid line represents the theoretical change in mesh size according to the explanation in the text. (f) The power law exponents of suspensions with respect to the MWCNT concentration.

region in the spectra at high $Q$ values (only seen in SAXS data at $Q>0.2$, not given here). The solid lines in Fig. 2(a-d) represent the best fit values according to eqn (1). ${ }^{22}$

The mesh size $\xi$ was determined by the crossover point of $Q^{-1}$ to $Q^{-2}$ power law behavior $\left(\xi=2 \pi / Q_{\mathrm{c}}\right)$ and the observed values for MWCNT suspensions in the epoxy $+\mathrm{BMIMBF}_{4}$ mixture are given in Fig. 2e. The value of $\xi$ decreases indicating that the nanotubes are getting close to each other with an increase in concentration of MWCNTs. Theoretically for cylindrical rods in semidilute concentration regimes the mesh size is proportional to the concentration $(c)$ of rods, i.e., $\xi \propto 1 / \sqrt{ } c .^{23}$ This relation is also shown by solid lines in Fig. 2e. From the figure, it is clear that the composites show less deviation from the theoretical values. The crossover behavior was observed for 
suspensions up to $0.3 \mathrm{wt} \%$ of MWCNTs however above this composition, a power law dependence on exponents having values higher than $1(\alpha>1)$ was observed. The value of $\alpha$ can be used to quantitatively determine the level of dispersion; a better dispersion can be assumed if $\alpha$ is close to 1 . Fig. $2 \mathrm{f}$ shows the $\alpha$ for different suspensions at varying MWCNT concentrations. The $\alpha$ increases with an increase in the nanotube concentrations which is attributed to the formation of MWCNT bundles and a wide distribution of size and structures. ${ }^{22}$ The results of epoxy suspensions containing $\mathrm{BMIMBF}_{4}$ were also compared with those without $\mathrm{BMIMBF}_{4}$ to see the level of dispersion where scattering intensity behavior with $\alpha>2$ was observed in all cases (not given here). This demonstrates the excellent capability of $\mathrm{BMIMBF}_{4}$ to disperse carbon nanotubes.

Epoxy composites containing $0.1-1 \mathrm{wt} \%$ of MWCNTs were prepared using the mixture 4,4'-methylenedianiline (MDA) $+\mathrm{BMIMBF}_{4}$ as a curing agent. The amount of $\mathrm{BMIMBF}_{4}$ was previously optimized to be $0.5 \%$ of epoxy for the best dispersion and mechanical performance and then a stoichiometric amount of MDA was employed (ESI $\dagger$ ). The efficacy of the $\mathrm{BMIMBF}_{4}$ in exfoliating and dispersing MWCNTs in epoxy composites is again demonstrated using SAXS data shown in Fig. 3a-c. The SAXS measurements were acquired on $25 \mathrm{~mm} \times 25 \mathrm{~mm} \times 2 \mathrm{~mm}$ composite strips using a $1 \mathrm{~mm}$ beam at $2 \mathrm{~mm}$ intervals. The scattering profiles of $1 \mathrm{wt} \%$ MWCNT composites are shown in Fig. $3 \mathrm{~b}$ and c. The SAXS profiles of composites prepared using $\mathrm{BMIMBF}_{4}$ as dispersing medium are identical at all positions of the strip (Fig. 3b) whereas those without ionic liquid differ both in intensity and scattering vector, $Q$-values (Fig. 3c).

The finely dispersed MWCNTs in the epoxy matrix can be observed in the high resolution scanning electron microscope
(SEM) image shown in Fig. 3d. The SEM images show dispersed and randomly oriented individual MWCNTs in $1 \mathrm{wt} \%$ composites. It can be suggested that due to the interaction between MWCNTs and ionic liquid, large nanotube aggregates would split into small segregates or individual tubes and become well dispersed in the epoxy matrix. To investigate the level of adhesion between the matrix and nanotubes, a comparison in the size of the individual nanotubes has been performed using the SEM images of composites prepared with and without $\mathrm{BMIMBF}_{4}$. It is surprising that the average diameter of nanotubes is $\sim 80 \mathrm{~nm}$ in composites where $\mathrm{BMIMBF}_{4}$ was added as a dispersing-curing agent. These individual nanotubes in the epoxy matrix are shown in Fig. 3e. The larger diameters observed suggest that the nanotubes were wrapped in or covered by a BMIMBF 4 + epoxy layer, indicating good adhesion between the matrix and MWCNTs. On the other hand, the average diameter of nanotubes was found to be around $50 \mathrm{~nm}$ in the case of composites prepared without $\mathrm{BMIMBF}_{4}$ (Fig. 3f). This is consistent with the diameter range of as-received MWCNTs as provided by the manufacturer (40-60 nm), indicating that there is no epoxy wetting or adhesion between MWCNTs and the matrix.

The composite samples were further examined using Raman spectroscopy (Fig. 4) to verify that the dispersion of MWCNTs using $\mathrm{BMIMBF}_{4}$ resulted in physical adsorption at the interface rather than disturbing the CNT structure to introduce defects. The two most distinguished features of CNTs in Raman spectra are the $\mathrm{D}$ and $\mathrm{G}$ bands observed at wave numbers of $\sim 1350 \mathrm{~cm}^{-1}$ and $\sim 1580 \mathrm{~cm}^{-1}$, respectively. The $\mathrm{G}$ band represents $\mathrm{sp}^{2}$ hybridized carbon networks and the $G$ band denotes the disorder-induced character. The integrated ratio of intensity
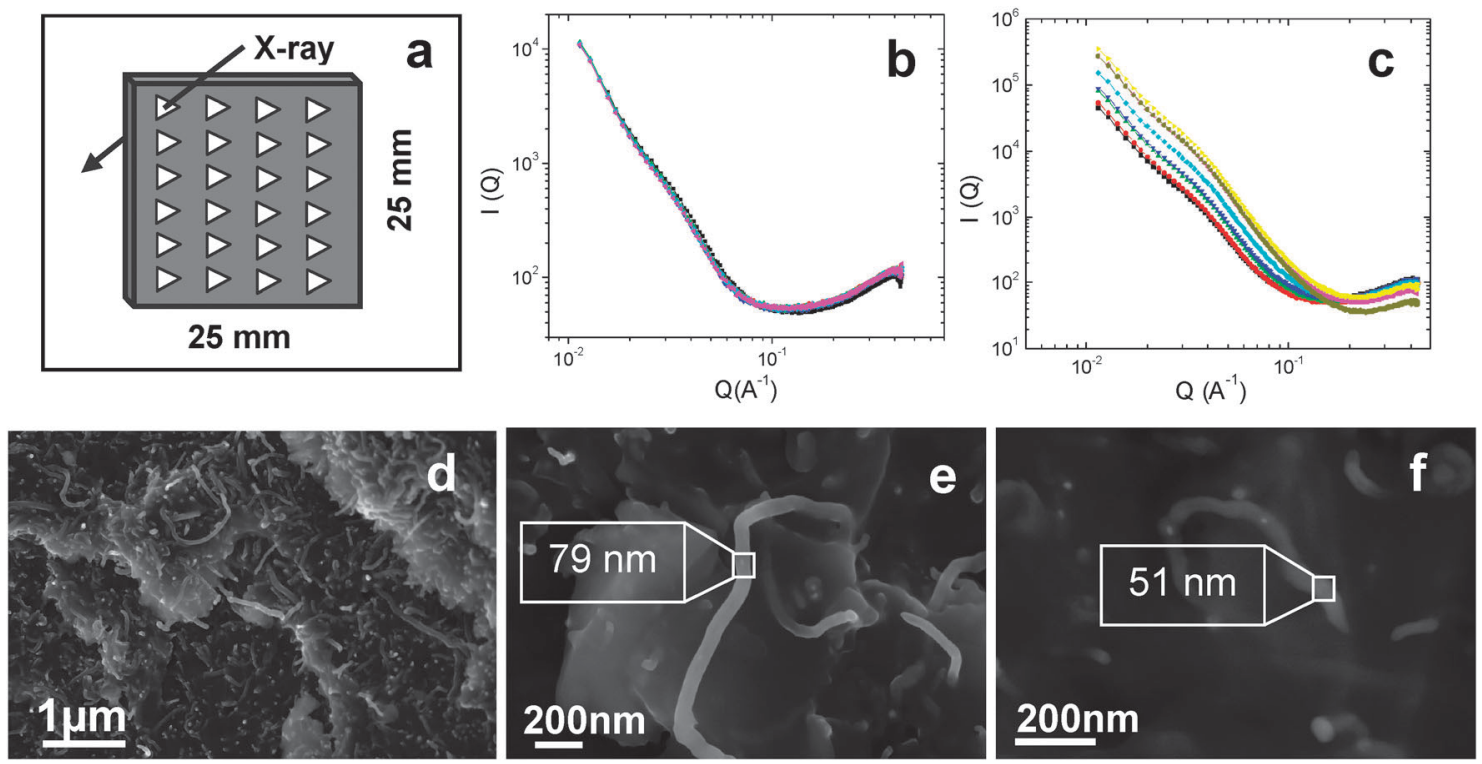

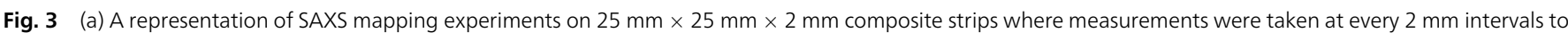

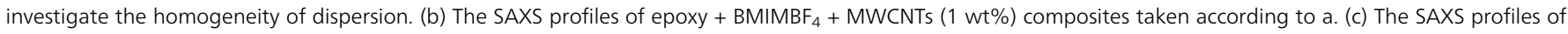

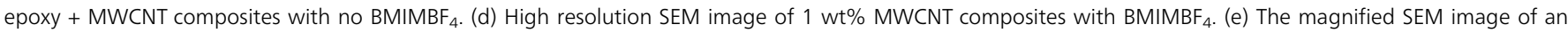

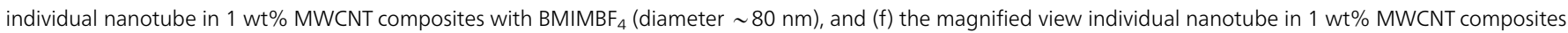
without $\mathrm{BMIMBF}_{4}$ where the diameter is only $\sim 50 \mathrm{~nm}$. 

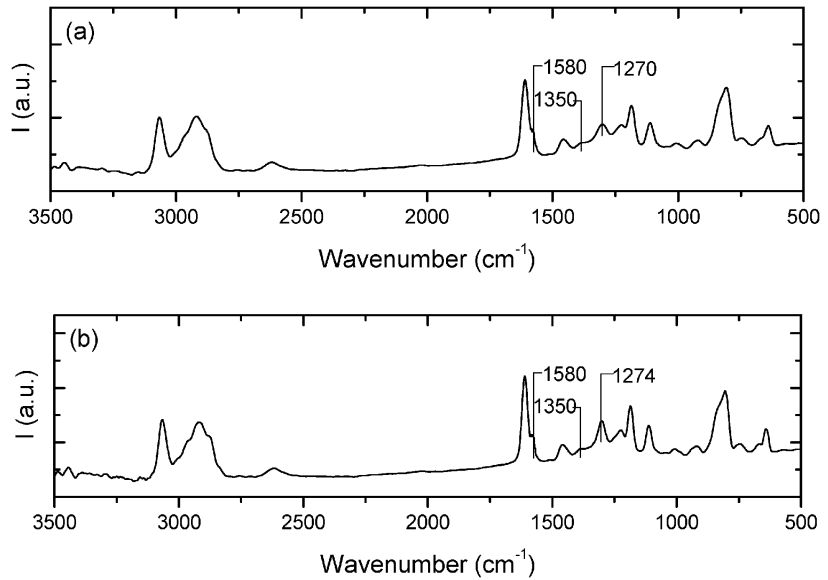

Fig. 4 Raman spectra of (a) epoxy + $\mathrm{BMIMBF}_{4}+\mathrm{MWCNTS}$ and (b) epoxy + MWCNT composites (1 wt\%) in the frequency range $3500-500 \mathrm{~cm}^{-1}$.

of these bands $\left(I_{\mathrm{D}} / I_{\mathrm{G}}\right)$ can be used as an indication of defect density of nanotubes. It can be seen that there is no significant changes in the position or intensity of D and G bands or the intensity ratio in samples with and without ionic liquid. This indicates that the dispersion of MWCNTs using $\mathrm{BMIMBF}_{4}$ was effective in epoxy without damaging nanotube walls. Moreover, the interaction between the matrix and MWCNTs can be verified by a peak width change in the spectra. ${ }^{24}$ It is observed that the peak at $1270 \mathrm{~cm}^{-1}$ is shifted to a higher wavenumber region $\left(1274 \mathrm{~cm}^{-1}\right)$ in composites containing IL and its width is narrower compared to the one without IL. This is due to the interaction of MWCNTs with surrounding polymer chains at the interface. ${ }^{25}$
The thermomechanical properties of neat epoxy and nanocomposites are presented in Fig. 5a-d. The glass transition temperature $\left(T_{\mathrm{g}}\right)$ and storage modulus of the different samples, at several temperatures, are summarized in Table 1 . The storage modulus of nanocomposites is remarkably higher than that of the neat epoxy at temperatures below $T_{\mathrm{g}}$ and then this value becomes similar for all the samples above $T_{\mathrm{g}}$. The incorporation of only $0.1 \mathrm{wt} \%$ of MWCNTs led to 33\% improvement in the storage modulus of glassy epoxy at $30{ }^{\circ} \mathrm{C}$ which is considered to be significant. The storage modulus value steadily increases with the amount of MWCNTs up to $1 \mathrm{wt} \%$ and becomes marginal above this concentration. All the composites showed higher $T_{\mathrm{g}}$ values compared to neat epoxy. The synergic behavior in $T_{\mathrm{g}}$ was again confirmed from differential scanning calorimetry (DSC) experiment results. Interestingly $T_{\mathrm{g}}$ of all composites show higher values, the maximum $T_{\mathrm{g}}$ was shown by $0.5 \mathrm{wt} \%$ composites $\left(\sim 182{ }^{\circ} \mathrm{C}\right)$ which is about 9 degree higher than the neat epoxy thermoset (Fig. S1 in the ESI $\dagger$ ). Generally there appears to be a reduction in $T_{\mathrm{g}}$ of epoxy upon the addition of any fillers. However, it was proven in our previous experiments that epoxy cured using the $\mathrm{BMIMBF}_{4}+$ MDA mixture shows an improvement in $T_{\mathrm{g}}$ by at least $8-10{ }^{\circ} \mathrm{C}$ than that cured with MDA alone (Table S1, ESI $\dagger$ ). Here, this synergic effect was retained even after the addition of MWCNTs. Moreover it was confirmed from thermal decomposition experiments that the thermal stability of the composites was increased marginally with the addition of MWCNTs (Fig. S2, ESI $\dagger$ ).

The fracture toughness was measured to investigate the effect of efficient nanotube dispersion on the fracture mechanical properties.


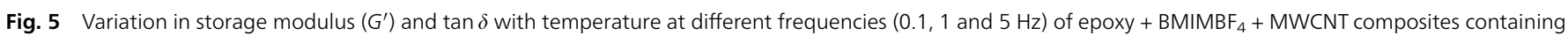
(a) 0 wt \%, (b) $0.1 \mathrm{wt} \%$ (c) $0.5 \mathrm{wt} \%$ and (d) $1 \mathrm{wt} \%$ MWCNTs. 
Table 1 The tensile mechanical and thermomechanical properties of composites prepared using $\mathrm{BMIMBF}_{4}$ as the dispersion-curing agent

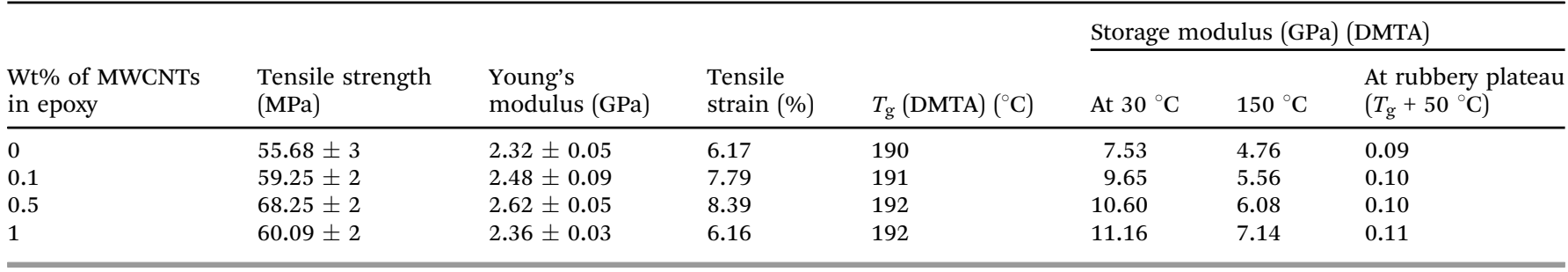

The plain-strain fracture toughness $\left(K_{1 \mathrm{C}}\right)$ and critical energy release rate $\left(G_{1 \mathrm{C}}\right)$ values of the neat epoxy and nanocomposites are summarized in Fig. 6a and b. It is clear that the resistance to fracture was significantly improved by the addition of MWCNTs. The largest improvement in $K_{1 \mathrm{C}}$ by $95 \%$ compared to neat epoxy was observed for $0.7 \mathrm{wt} \%$ MWCNT composites. The increase in fracture toughness can be attributed to the good level of MWCNT dispersion in IL up to $0.7 \mathrm{wt} \%$ concentration. This improvement is significant considering the fact that the total loading of CNT is only $0.7 \mathrm{wt} \%$ and the epoxy has a high cross-link density.

Generally the increase in toughness is at the expense of tensile strength and Young's modulus in the case of epoxy blends and composites. However, here the tensile properties of the composites are also increased at low MWCNT addition as shown in Table 1. It can be suggested that $\mathrm{BMIMBF}_{4}$ addition in epoxy composites has led to a higher interfacial bonding. The SEM images of the fractured surface at high magnification of composites with and without ionic liquid have been compared. The images are shown in Fig. $6 \mathrm{c}$ and d respectively. In the case of composites without ionic liquid, nanotubes are pulled out from the matrix and randomly seen on the surface that underlines a poor adhesion. Although with ionic liquid $\mathrm{BMIMBF}_{4}$ as a dispersion-curing agent, nanotubes break at fracture instead of slipping and appear to be projecting out of the fracture surface which indicates a good interfacial adhesion. It is difficult to ascertain the mechanism of fracture toughness improvement at this point; however, a possible assumption is that the formation of a unique epoxy network structure with a definite asymmetric distribution of cross-links around the carbon nanotubes enhances the ductility of the matrix.

The thermomechanical and fracture properties of the composites prepared using IL have been compared with existing epoxy-CNT composites prepared via other routes and are shown in Table S2 (ESI + ). The most common methods involve the use of solvents, surfactants or block copolymers as dispersing agents for CNTs or functionalization of CNTs. Most of the studies reported an unchanged $T_{\mathrm{g}}$ or a reduction in $T_{\mathrm{g}}$ occurs by the incorporation of nanotubes into the matrix. ${ }^{26-34}$ From Table S2 (ESI $)$, it is clear that IL is the simple and effective dispersioncuring agent that can significantly improve the toughness of the matrix without deteriorating other physical properties.


Fig. 6 (a) $K_{1 C}$ and (b) $G_{1 C}$ fracture properties of the epoxy composites with different MWCNT contents. (c) SEM fracture images of composites without BMIMBF 4 dispersion. Individual nanotubes are randomly distributed on the fractured surface showing no interfacial compatibility between the matrix and the nanotubes. (d) Composites with $\mathrm{BMIMBF}_{4}$ as the dispersion-curing agent. MWCNTs are dispersed well within the matrix showing excellent adhesion. 
This is obviously due to the high level of nanotube dispersion achieved in IL as proven by the scattering studies.

\section{Conclusions}

The dispersion-curing method using ionic liquid outlined here allows the fabrication of highly dispersed nanotubes/epoxy suspensions and nanocomposites with superior properties. The role of the ionic liquid is to function as a dispersing agent prior to curing and later as a curing agent. It can be assumed that the addition of the ionic liquid also has an effect on the wetting behavior, and on the interfacial adhesion, which in return has an impact on the ionic liquid's ability to exfoliate the nanotubes. The composites at low nanotube loading showed exceptionally high fracture mechanical strength and modulus without reduction in tensile properties. The interfacial chemistry of carbon nanotubes with the ionic liquid and epoxy should be an important subject for future research.

\section{Experimental section}

Multiwalled carbon nanotubes (MWCNTs) were obtained from Shenzhen Nano-Tech Co Ltd. The nanotubes were with a diameter of 40-60 $\mathrm{nm}$ and a length of 1-2 $\mu \mathrm{m}$. The epoxy precursor diglycidyl ether of bisphenol A (DGEBA) with epoxide equivalent weight of 172-176 and the curing agent 4,4'-methylenedianiline (MDA) were purchased from Aldrich Chemical Co. The ionic liquid 1-butyl-3-methylimidazolium tetrafluoroborate $\left(\mathrm{BMIMBF}_{4}\right)$ was from Merck. The chemicals were used as received to simplify the process.

For USAXS measurements the MWCNTs were suspended in a solution of the epoxy $+\mathrm{BMIMBF}_{4}$ mixture, sonicated for 1 hour and then mechanically stirred for 2 hours. For the fabrication of epoxy composites a method based on the mixing of MWCNTs and resin was used. Pre-weighed amounts of MWCNTs were first suspended in the epoxy $+\mathrm{BMIMBF}_{4}$ mixture and then sonicated for 1 hour. The suspension was then mechanically stirred for 2 hours using an Ika Werke RW16 high speed mixer at $1200 \mathrm{rpm}$ and then MDA was added. After complete dissolution of MDA, the whole mixture was poured into pre-heated moulds and cured at $150{ }^{\circ} \mathrm{C}$ for 10 hours and then post-cured at $180{ }^{\circ} \mathrm{C}$ for 2 hours.

USAXS experiments were performed at the Australian Synchrotron on the ultra-small and small angle X-ray scattering beamline utilizing an undulator source that allows measurement at a very high flux to moderate scattering angles and a good flux at the minimum $q$ limit. The experiments were conducted at room temperature and the wavelength of X-ray was $0.062 \mathrm{~nm}$. The intensity profiles were interpreted from the plot of scattering intensity $(I)$ versus scattering vector, $q=(4 \pi / \lambda) \sin (\theta / 2)(\theta$ is the scattering angle). Raman spectra were recorded with a Perkin Elmer Raman station 400 spectrometer using Helium laser excitation. The samples were scanned from $400 \mathrm{~cm}^{-1}$ to $3624 \mathrm{~cm}^{-1}$ at a resolution of $4 \mathrm{~cm}^{-1}$. Dynamic mechanical tests were performed on a dynamic mechanical thermal analyzer (DMTA) (TA Q800, USA) in a cantilever mode.
The frequency was $1 \mathrm{~Hz}$ at a heating rate of $3{ }^{\circ} \mathrm{C} \mathrm{min}{ }^{-1}$. The specimen dimensions were $60 \times 12 \times 3 \mathrm{~mm}^{3}$. The storage modulus, loss modulus and $\tan \delta$ were measured from 30 to $250{ }^{\circ} \mathrm{C}$. The $T_{\mathrm{g}}$ was taken as the maximum of the $\tan \delta$ curve in the glass transition region. For fracture toughness measurements, the single-edge-notch three point-bending (SENB) test was used according to ASTM standard D5045. The tests were performed on an Instron $30 \mathrm{kN}$ instrument at a test speed of $2 \mathrm{~mm} \mathrm{~min}^{-1}$. An initial crack was generated carefully by tapping the thumbnail shape crack front using a fresh razor blade prior to testing. $K_{1 \mathrm{C}}$ and $G_{1 \mathrm{C}}$ of the samples were determined by testing five specimens. The tensile properties of the composites were measured on an Instron $30 \mathrm{kN} \mathrm{SD}$ tensile testing machine. The tests were conducted with dumbbell shaped specimens according to ASTM standard D638. At least five specimens were tested to obtain the average values of tensile properties for all the specimens.

\section{Acknowledgements}

SAXS/WAXS research was undertaken at the Australian Synchrotron, Victoria, Australia, and the authors thank Dr Nigel Kirby and Dr Adrian Hawley for their assistance. The authors thank Dr Joel Davis at Australian Nuclear Science and Technology Organisation (ANSTO) for SEM imaging. The authors thank Dr Muthu Pannirselvam at the Rheology and Materials Processing Centre (RMPC), RMIT University for Raman experiments. We also thank the Australian Institute of Nuclear Science and Engineering (AINSE) Ltd for an award (ALNGRA 11157) for the SAXS measurements.

\section{References}

1 S. M. Liff, N. Kumar and G. H. McKinley, Nat. Mater., 2007, 6, 76.

2 P. M. Ajayan, L. S. Schadler, C. Giannaris and A. Rubio, Adv. Mater., 2000, 12, 750.

3 P.-C. Ma, S.-Y. Mo, B.-Z. Tang and J.-K. Kim, Carbon, 2010, 48, 1824.

4 L. Schadler, Appl. Phys. Lett., 1998, 73, 3842.

5 J. Suhr, N. Koratkar, P. Keblinski and P. Ajayan, Nat. Mater., 2005, 4, 134.

6 L. Jeongwoo, K. Myunghun, K. H. Chang and E. S. Sang, Meas. Sci. Technol., 2007, 18, 3707.

7 J. Zhu, J. Kim, H. Peng, J. L. Margrave, V. N. Khabashesku and E. V. Barrera, Nano Lett., 2003, 3, 1107.

8 J. Zhu, H. Peng, F. Rodriguez-Macias, J. L. Margrave, V. N. Khabashesku, A. M. Imam, K. Lozano and E. V. Barrera, Adv. Funct. Mater., 2004, 14, 643.

9 O. Osazuwa, K. Petrie, M. Kontopoulou, P. Xiang, Z. Ye and A. Docoslis, Compos. Sci. Technol., 2012, 73, 27.

10 T. Fukushima, A. Kosaka, Y. Ishimura, T. Yamamoto, T. Takigawa, N. Ishii and T. Aida, Science, 2003, 300, 2072.

11 J. C. Ma and D. A. Dougherty, Chem. Rev., 1997, 97, 1303.

12 J. Wang, H. Chu and Y. Li, ACS Nano, 2008, 2, 2540. 
13 M. Zheng, A. Jagota, E. D. Semke, B. A. Diner, R. S. McLean, S. R. Lustig, R. E. Richardson and N. G. Tassi, Nat. Mater., 2003, 2, 338.

$14 \mathrm{~K}$. Krzysztof and S. Tadeusz, Ionic liquids as convenient latent hardeners of epoxy resins: Rapid communications, Industrial Chemistry Research Institute, Warszawa, POLOGNE, 2003, p. 48.

15 M. A. M. Rahmathullah, A. Jeyarajasingam, B. Merritt, M. VanLandingham, S. H. McKnight and G. R. Palmese, Macromolecules, 2009, 42, 3219.

16 H. Zhang, Z. Wang, Z. Zhang, J. Wu, J. Zhang and J. He, Adv. Mater., 2007, 19, 698.

17 Z. Wang, X. Yang, Q. Wang, H. T. Hahn, S. Lee, K. H. Lee and Z. Guo, Int. J. Smart Nano Mater., 2011, 2, 176.

18 Z. Wang, H. A. Colorad, Z. H. Guo, H. Kim, C. L. Park, H. T. Hahn, S. G. Lee, K. H. Lee and Y. Q. Shang, J. Mater. Res., 2012, 15, 510.

19 L. A. Hough, M. F. Islam, B. Hammouda, A. G. Yodh and P. A. Heiney, Nano Lett., 2006, 6, 313.

20 W. Zhou, Chem. Phys. Lett., 2004, 384, 185.

21 K. Yurekli, C. A. Mitchell and R. Krishnamoorti, J. Am. Chem. Soc., 2004, 126, 9902.

22 H. Wang, W. Zhou, D. L. Ho, K. I. Winey, J. E. Fischer, C. J. Glinka and E. K. Hobbie, Nano Lett., 2004, 4, 1789.

23 C. F. Schmidt, M. Baermann, G. Isenberg and E. Sackmann, Macromolecules, 1989, 22, 3638.
24 Q. Zhao and H. D. Wagner, Philos. Trans. R. Soc., A, 2004, 362, 2407.

25 L. Valentini, J. Biagiotti, J. M. Kenny and S. Santucci, Compos. Sci. Techol., 2003, 63, 1149.

26 M. R. Loos, J. Yang, D. L. Feke and I. Manas-Zloczower, Compos. Sci. Techol., 2012, 72, 482.

27 L. Guadagno, B. De Vivo, A. Di Bartolomeo, P. Lamberti, A. Sorrentino, V. Tucci, L. Vertuccio and V. Vittoria, Carbon, 2011, 49, 1919.

28 L. C. Tang, H. Zhang, J. Han, X. Wu and Z. Zhang, Compos. Sci. Techol., 2011, 72, 7.

29 G. Gkikas, N.-M. Barkoula and A. S. Paipetis, Composites, Part B, 2012, 43, 2697.

30 J. Shen, W. Huang, L. Wu, Y. Hu and M. Ye, Composites, Part A, 2007, 38, 1331.

31 F. H. Gojny, M. H. G. Wichmann, B. Fiedler and K. Schulte, Compos. Sci. Techol., 2005, 65, 2300.

32 R. Hollertz, S. Chatterjee, H. Gutmann, T. Geiger, F. A. Nuesch and B. T. T. Chu, Nanotechnology, 2011, 22, 125702.

33 L. Sun, G. L. Warren, J. Y. O'Reilly, W. N. Everett, S. M. Lee, D. Davis, D. Lagoudas and H.-J. Sue, Carbon, 2008, 46, 320.

34 S. Cuia, R. Caneta, A. Derrea, M. Couzib and P. Delhaes, Carbon, 2003, 41, 797. 